\title{
Does Mindful Parenting Mediate the Association between Maternal Anxiety during Pregnancy and Child Behavioral/Emotional Problems?
}

\author{
Jens Henrichs ${ }^{1}$ - Marion I. van den Heuvel $^{2}$ - Anke B. Witteveen ${ }^{1}$ • Janneke Wilschut ${ }^{3}$ • Bea R. H. Van den Bergh ${ }^{4,5}$
}

Published online: 26 February 2019

(C) The Author(s) 2019

\begin{abstract}
Objectives Maternal anxiety during pregnancy is a risk factor for child behavioral/emotional problems. Knowledge about explanatory factors mediating this link is scarce. Maternal anxiety during pregnancy may be an indicator of adverse postnatal environmental conditions, including maternal anxiety and poor parenting, possibly affecting child behavior. This study investigated whether maternal anxiety and mindful parenting in early childhood mediate the association between maternal anxiety during pregnancy and child behavioral/emotional problems.

Methods This study was based on a sample of 118 mother-child dyads who participated in a cohort study that followed participants from pregnancy until 4 years after birth. At 21 weeks' gestation and when the child was 4 years old, mothers completed questionnaires to assess state anxiety (State-Trait Anxiety Inventory) and general anxiety (Symptom Checklist-90 anxiety subscale). At age 4 years, mothers reported mindful parenting (Interpersonal Mindfulness in Parenting Scale) and child behavioral/emotional problems (Child Behavior Checklist).

Results Maternal state anxiety during pregnancy was positively associated with child internalizing problems and negatively with mindful parenting. Maternal general anxiety during pregnancy was related with more child internalizing and externalizing problems but not with mindful parenting. The association between maternal state anxiety during pregnancy and child internalizing problems was sequentially mediated via concurrent maternal general anxiety and mindful parenting.

Conclusions These findings suggest that maternal anxiety during pregnancy continues into early childhood and, because of this, it subsequently affects child internalizing behavior via poor mindful parenting. Replication studies are needed before developing interventions for tackling maternal anxiety during pregnancy and promoting mindful parenting.
\end{abstract}

Keywords Mindful parenting $\cdot$ Maternal anxiety $\cdot$ Child behavioral/emotional problems $\cdot$ Mediation $\cdot$ Pregnancy

Marion I. van den Heuvel

m.i.vdnheuvel@tilburguniversity.edu

1 Amsterdam UMC, Vrije Universiteit Amsterdam, Department of Midwifery Science, AVAG-Amsterdam Public Health, Van der Boechorststraat 7, 1081, BT Amsterdam, the Netherlands

2 Department of Cognitive Neuropsychology, Tilburg University, PO Box 90153, LE Tilburg, the Netherlands

3 Amsterdam UMC, Vrije Universiteit Amsterdam, Department of Epidemiology and Biostatistics, Amsterdam Public Health, Van der Boechorststraat 7, 1081, BT Amsterdam, the Netherlands

4 Health Psychology, Faculty of Psychology and Educational Sciences, KU Leuven-University of Leuven, Tienesestraat 102, PO Box 3726, 3000 Leuven, Belgium

5 Department of Welfare, Public Health and Family, Flemish Government, Koning Albert-II laan 35, 1030 Brussels, Belgium
Emerging evidence demonstrates that maternal anxiety during pregnancy is a risk factor for offspring behavioral/emotional problems (i.e., internalizing and externalizing problems) in childhood and adolescence and even for psychopathology in adulthood (Achenbach and Rescorla 2000; Stein et al. 2014; Talge et al. 2007; Van den Bergh et al. 2017). This indicates that maternal anxiety during pregnancy is a serious public health problem, which seems to contribute to the continuities of psychopathology from early childhood into adulthood (Hofstra et al. 2000; Reef et al. 2010). Animal models, natural experiments, and other empirical research in humans all suggest that maternal anxiety/stress during pregnancy can affect fetal neurodevelopment and subsequent child behavioral outcomes via intrauterine mechanisms, including maternal stress hormones and epigenetic alterations (Beijers et al. 2014; Sandman et al. 2011; Van den Bergh et al. 2017). A review 
of human studies by Sandman et al. (2011) indicated that fetal exposure to elevated levels of maternal stress hormones was linked to child behavioral/emotional problems, independent of birth outcomes and maternal postnatal depression or stress. However, postnatal environmental conditions may also mediate the link between maternal anxiety during pregnancy and child behavioral/emotional outcome (Stein et al. 2014). Maternal anxiety during pregnancy may be an early indicator of poor postnatal environmental conditions - including poor parenting and maternal postnatal anxiety - that may negatively affect child behavioral development (Beijers et al. 2014).

Studying the role of both maternal anxiety in early childhood and parenting abilities could provide crucial information for developing and optimizing interventions aimed at promoting child behavioral development from early life onwards. Research addressing the mediating role of parenting abilities in this link is sparse. So far, only a small number of studies have shown that parenting abilities (i.e., positive discipline and maternal sensitivity) mediate the association of prenatal maternal depressive symptoms/stress with child behavioral outcomes in early childhood (Edwards and Hans 2016; Kok et al. 2013). The mediating role of parenting abilities in the link between maternal anxiety during pregnancy and child behavioral/emotional outcomes has, however, not been studied so far. Although anxiety shares a common component with stress and depression (i.e., general distress), it is also characterized by unique clinical features and symptomatology (e.g., physiological hyperarousal) (Clark and Watson 1991; Nieuwenhuijsen et al. 2003). Research into the links between maternal anxiety in the prenatal and early childhood period, parenting abilities, and child behavioral/emotional problems fills knowledge gaps regarding how these environmental factors shape child behavioral functioning.

Stein et al. (2014) proposed that the effects of maternal anxiety during pregnancy on the child are mediated by exposure to persisting maternal anxiety later in life. Indeed, maternal anxiety during pregnancy and in early childhood and child behavioral/emotional problems are interrelated (O'Connor et al. 2002; van Batenburg-Eddes et al. 2013). Maternal anxiety at 20 weeks' gestation predicts maternal anxiety at age 3 years (van Batenburg-Eddes et al. 2013). Based on the population-based ALSPAC cohort, O'Connor et al. (2002) reported that maternal anxiety at 21 months postpartum predicted child attention problems at age 47 months. A crosscohort consistency study found that maternal anxiety at age 3 years partly explained the association between maternal anxiety at 18 or 20 weeks' gestation, respectively, and child emotional problems at age 3 and 4 years, but that it completely explained the association between prenatal maternal anxiety and child attention problems (van Batenburg-Eddes et al. 2013). Interestingly, Stein et al. (2014) suggested that maternal anxiety in pregnancy and early childhood can also impair parenting abilities.
The basis for early parenting abilities starts to evolve during pregnancy making these abilities vulnerable for adverse prenatal psychosocial influences, including maternal anxiety. The attachment theory posits that, from pregnancy onwards, parents develop internal representations of their (unborn) child based on both their own attachment relationship experiences in childhood and present contextual factors, including partner relationship quality and anxiety (Dayton et al. 2010). These internal representations guide parenting behaviors (Dayton et al. 2010). The mother-child relationship forms the foundation for child socio-emotional and behavioral development. Poor motherchild relationships and poor parenting abilities are both associated with child behavioral/emotional problems (Bögels et al. 2010; Stein et al. 2014). One crucial part of the early motherchild relationship concerns the development of the emotional tie of the mother with her unborn child (i.e., maternal bonding), which starts to evolve during pregnancy (Brandon et al. 2009; Hicks et al. 2018). Prenatally formed bonding patterns continue into the postpartum period (de Cock et al. 2016). Perinatal bonding patterns are associated with parenting quality, the quality of mother-child interactions, and infant-mother attachment (Brandon et al. 2009; Maas et al. 2016; Van den Bergh and Simons 2009). Maternal anxiety and depression symptoms, which often co-occur, can hamper the development of the prenatal bond, however, and these factors can subsequently disturb the development of parenting abilities (Brandon et al. 2009; Henrichs et al. 2009; Pearson et al. 2012). Previous work suggests that maternal anxiety negatively influences parenting via altered parental cognitions, emotions, and behavior, including rumination and worry (Choi et al. 2010; Stein et al. 2012, 2014). Expectant mothers with depression symptoms sometimes experience problems in feeling a connection with the fetus, experience strong fears about harmful effects on the baby, may feel inadequate about their abilities as caregiver, and display low maternal responsiveness during mother-infant interactions at age 12 months (Junge et al. 2017; Pearson et al. 2012). From pregnancy onwards, maternal anxiety may thus affect (the development of parenting abilities like maternal responsiveness, which is a crucial component of mindful parenting (Duncan et al. 2009).

Mindful parenting may concern a parenting ability involved in the link between maternal anxiety during pregnancy with child behavioral/emotional problems. Mindful parenting is a relevant parenting ability and has lately received increased attention by researchers. Siegel and Hartzell (2003) suggested that mindful parenting represents one way how to promote the quality of the child-mother attachment relationship, which starts to develop early in life and is vulnerable for parental anxiety (Brandon et al. 2009; Dayton et al. 2010). It has been posited that mindful parenting can help both parents and children to regulate distress (Laurent et al. 2017). In their conceptual model, Duncan et al. (2009) defined mindful parenting as parenting practices that positively influence the parent-child relationship, including listening 
with full attention, non-judgmental acceptance, emotional awareness (including parental responsiveness), compassion for self and child, and self-regulation in the parenting relationship. These practices can positively influence parental well-being, parentchild affection, and parenting practices, in turn leading to a reduction of child behavioral/emotional problems and to higher levels of child well-being (Duncan et al. 2009). Indeed, mindful parenting is negatively related to dysfunctional parenting styles and, notably, also to parental anxiety (de Bruin et al. 2014; Geurtzen et al. 2015). However, mindful parenting 3 months postpartum predicted better stress axis activity (i.e., lower cortisol levels) among 6-month-old infants of mothers with high life stress (Laurent et al. 2017). Mindful parenting is also associated with less internalizing and externalizing problems in young children, schoolchildren, and adolescents (Geurtzen et al. 2015; Parent et al. 2016).

Sex differences in behavioral/emotional problems start to emerge during early childhood. In a population-based study, girls aged 3 years had higher levels of parent-reported internalizing problems than boys, while boys had more externalizing problems (Henrichs et al. 2013). Child sex has been shown to moderate the associations between maternal anxiety or negative parenting and child outcomes (Barnett and Scaramella 2013; Stein et al. 2014). More negative parenting behaviors were related to more externalizing problems for boys, while less positive parenting behaviors were related to more externalizing problems for girls (Barnett and Scaramella 2013).

Using a prospective cohort study, we examined whether maternal anxiety in early childhood and mindful parenting independently and/or sequentially mediate the possible association between maternal anxiety during pregnancy and child behavioral/emotional problems at age 4 years. Importantly, anxiety comprises different types of anxiety symptoms (Gelenberg 2000). To be able to assess whether different types of anxiety symptoms are associated with child behavioral/emotional problems in different ways, we used self-report measures of both state and general anxiety within a prospective cohort study. Whereas state anxiety covers the mental symptoms of anxiety due to momentary life stress (Spielberger 1975), general anxiety mainly comprises somatic anxiety symptoms rather than mental ones (Derogatis et al. 1974). Additionally, we investigated whether the mediation effects of maternal anxiety and mindful parenting in early childhood on the association between maternal anxiety during pregnancy and child behavioral/emotional problems were moderated by child sex.

\section{Method}

\section{Participants}

Participants were enrolled in a prospective cohort study, the Prenatal Early Life Stress (PELS) Project, following children and their parents from fetal life onwards. Participating mothers $(N=190)$ were enrolled between the 9th-15th week of gestation $(n=178)$ or between the 16th and 23rd week of gestation $(n=12)$ via four midwifery practices and one general hospital in Tilburg, The Netherlands. Data on maternal anxiety during pregnancy were available in 171 eligible subjects (89\% of 190 initially enrolled subjects). At the 4-year follow-up, 53 subjects had no data available on concurrent maternal anxiety, mindful parenting, child characteristics, and/or child outcomes due to, for example, drop-out, loss-to-follow-up, or not returning questionnaires. This left up to 118 mother-child dyads $(69 \%$ of 171 eligible subjects) that were included in our main analyses. Mothers $(n=118)$ reported maternal anxiety at 21 weeks' gestation $\left(M_{\text {weeks }}=20.8\right.$ weeks, $\left.S D=1.8\right)$ and maternal anxiety, mindful parenting, and child behavioral/emotional problems at age 4 years $\left(M_{\text {months }}=48.1, S D=0.9\right)$. Table 1 presents sample characteristics of participating mothers and children.

To examine whether non-response was selective, we compared the core data of included children (who had data on prenatal maternal anxiety, concurrent maternal anxiety, mindful parenting, and child outcomes at age 4 years) with that of eligible children who were excluded due to missing data on mindful parenting, concurrent maternal anxiety, and child outcomes. Between included and excluded children, there were no significant differences in birth weight, $M=3422 \mathrm{~g}$, $S D=479$, vs. $3474 \mathrm{~g}, S D=491, t(104)=0.65, p=.52$, and gestational age, $M_{\text {weeks }}=39.7, S D=1.4$ vs. $M_{\text {weeks }}=39.8$, $S D=1.7, t(110)=0.31, p=.76$. Mothers of included children did, however, have significantly lower levels of prenatal state and general anxiety than mothers of excluded children, e.g., $M_{\text {state anxiety }}=31.2, S D=7.1 \mathrm{vs}$. $M_{\text {state anxiety }}=34.4, S D=9.2$, $t(117)=2.49, p=0.014$.

\section{Procedures}

Participating mothers were enrolled during the first or second trimester of pregnancy. Mothers filled out questionnaires at three time points during pregnancy (each trimester; T1, T2, T3). After birth, mother and infant were invited to the laboratory at Tilburg University at 2 (T4) and 9 months (T5) after birth and when the child was 4 years old (T6). Mothers completed questionnaires at home around the same time as the laboratory visit. Questionnaires were either digitally or with pen and paper, depending on the preference of the mother. Qualtrics software (Qualtrics, Provo, UT; https://www. qualtrics.com) was used for the development and administration of the online questionnaires. Only data from T2, T4, and T6 were used for the current study.

\section{Measures}

Maternal State Anxiety At 21 weeks' gestation and at age 4 years, mothers reported state anxiety. State anxiety was 
Table 1 Sample characteristics $(N=118)$

\begin{tabular}{|c|c|c|}
\hline Maternal characteristics & $N^{\mathrm{a}}$ & $M(S D)^{\mathrm{a}}$ \\
\hline Age at enrollment, years & 118 & $32.0(3.8)$ \\
\hline \multicolumn{3}{|l|}{ Educational level (\%) } \\
\hline $\operatorname{High}^{\mathrm{b}}$ & 83 & 70.3 \\
\hline Low/medium ${ }^{\mathrm{b}}$ & 35 & 29.7 \\
\hline \multicolumn{3}{|l|}{ Ethnicity } \\
\hline Dutch & 113 & 95.8 \\
\hline Non-Dutch & 5 & 4.2 \\
\hline \multicolumn{3}{|l|}{ Family income } \\
\hline High (i.e. >€3600) & 92 & 78.0 \\
\hline (low/below) average ${ }^{\mathrm{c}}$ & 18 & 15.3 \\
\hline Missing & 8 & 6.8 \\
\hline \multicolumn{3}{|l|}{ Marital status } \\
\hline Married & 72 & 61.0 \\
\hline Living together & 46 & 39.0 \\
\hline \multicolumn{3}{|l|}{ Parity $^{\mathrm{d}}(\%)$} \\
\hline 0 & 49 & 41.5 \\
\hline$\geq 1$ & 64 & 54.2 \\
\hline Missing & 5 & 4.2 \\
\hline \multicolumn{3}{|l|}{ Smoking during pregnancy (\%) } \\
\hline Yes & 3 & 2.5 \\
\hline No & 113 & 95.8 \\
\hline Missing & 2 & 1.7 \\
\hline \multicolumn{3}{|l|}{ Alcohol use during pregnancy } \\
\hline Yes & 10 & 8.5 \\
\hline No & 105 & 89.0 \\
\hline Missing & 3 & 2.5 \\
\hline \multicolumn{3}{|l|}{ Child characteristics } \\
\hline Gestational age at birth, weeks ${ }^{\mathrm{e}}$ & 111 & $39.7(1.4)$ \\
\hline Child age 4-year follow-up, months ${ }^{\mathrm{e}}$ & 105 & $48.1(0.9)$ \\
\hline \multicolumn{3}{|l|}{$\operatorname{Sex}(\%)$} \\
\hline Boys & 56 & 47.5 \\
\hline Girls & 62 & 52.5 \\
\hline Birth weight, $\mathrm{g}^{\mathrm{e}}$ & 114 & $3422(479)$ \\
\hline Infant negative affectivity at age 3 months ${ }^{\mathrm{e}}$ & 90 & $34.3(11.3)$ \\
\hline
\end{tabular}

${ }^{\text {a }}$ Numbers differ due to missing values and represent means $(S D)$ unless otherwise indicated

${ }^{\mathrm{b}}$ Low/medium educational level = lower vocational training or less; high educational level $=$ at least higher vocational training or a bachelor's degree

${ }^{\mathrm{c}}$ (Low/below) average income comprises three categories of family income per month due to small numbers: $<€ 1500(n=1,0.8 \%), € 1600$ to $€ 2100$ ( $n=2,1.7 \%)$; $€ 2200$ to $€ 3500(n=15,12.7 \%)$

${ }^{\mathrm{d}}$ Parity indicates the number of times that women gave birth to a fetus with a viable gestational age

${ }^{\mathrm{e}}$ The following continuous variables had missing values: gestational age (5.9\%), birth weight (3.4\%), child age at 4-year follow-up (11.0\%), and infant negativity at age 3 months $(23.7 \%)$ assessed using the 20-item state anxiety subscale of the Dutch validated version of the State-Trait Anxiety Inventory (STAI) (Van der Ploeg et al. 1980). This subscale covers mental anxiety symptoms and measures the intensity of state anxiety in response to current life stress (Spielberger 1975). Mothers were asked to indicate how well each statement, such as "I am worried", of this subscale reflected their current emotional state $(1=$ not at all, $4=$ very much so $)$. Total subscale sum scores were calculated with higher scores indicating more state anxiety. The STAI-state subscale has good validity (Van der Ploeg et al. 1980). In our study, it had good internal consistency at 21 weeks' gestation and at age 4 years $(\alpha=.89$ and $\alpha=.94$, respectively). In our study, rates of high prenatal state anxiety (i.e., state subscale scores $\geq 43$ as defined at 21 weeks' gestation) were low (i.e., 7.6\%), as compared to the rates of high state anxiety $(30.9 \%)$ during the first half of pregnancy in a Dutch population-based study $(n=6443)$ (Koelewijn et al. 2017).

Maternal General Anxiety Mothers reported general anxiety using the validated Dutch version of the anxiety subscale of the Symptoms-Checklist-90 (SCL-90) (Arrindell and Ettema 2003). This subscale measures a cluster of psychiatric symptoms of general anxiety mainly covering somatic anxiety symptoms and vegetative arousal but assessing mental and general anxiety symptoms to a lesser degree (Arrindell and Ettema 2003; Derogatis et al. 1974). Women rated this 10item subscale by answering items such as "Nervousness or shaking inside" ( $1=$ not at all, $5=$ extremely). We calculated total subscale sum scores. Higher scores reflect increased general anxiety. The subscale has good validity (Arrindell and Ettema 2003) and showed adequate internal consistency at 21 weeks' gestation and at age 4 years $(\alpha=.76$ and $\alpha=.90$, respectively). Mean general anxiety scores at 21 weeks' gestation, $M_{\text {weeks }}=12.9, S D=3.2$, were comparable to mean general anxiety at a similar time point in pregnancy, $M_{\text {weeks }}=$ $12.3, S D=3.6$, of a Dutch population-based pregnancy sample $(n=845)$ (Bergink et al. 2011).

Mindful Parenting At age 4 years, mothers reported mindful parenting using the validated Dutch version of the Interpersonal Mindfulness in Parenting Scale (IM-P-NL) (de Bruin et al. 2014). The IM-P-NL consists of 29 items such as "React too quickly to child" ( $1=$ never true, $5=$ always true $)$. We calculated total IMP-NL sum scores. Higher IM-P-NL sum scores reflect more mindfulness in parenting. The IMP-NL has good validity (de Bruin et al. 2014) and showed good internal consistency, $\alpha=.86$, in our study.

Child Behavior Checklist 11/2-5 (CBCL 11/2-5) Mothers completed the CBCL/1.5-5 at age 4 years (Achenbach and Rescorla 2000). Respondents were asked to rate each of the 99 problems referring to the last 2 months, such as 
"worries", on a 3 -point Likert scale $(0=$ not true, $2=$ very true or often true). Two broad-band scales based on second-order factor-analyses of the factoranalytically established syndromes were used in our study: (1) internalizing, which is the sum of scores on the emotionally reactive, anxious/depressed, somatic complaints, and withdrawn syndromes, and (2) externalizing, which is the sum of scores on the attention problems and aggressive behavior syndromes. Test-retest reliability for the CBCL/1.5-5 ranged from 0.68 to 0.92 for syndrome scales and construct validity from 0.59 to 0.89 (Achenbach and Rescorla 2000). For the internalizing and externalizing scale, sum scores and T-scores were calculated. In our study, internal consistencies were good for the internalizing scale, $\alpha=0.84$, and the externalizing scale, $\alpha=0.87$. Children had similar mean internalizing $(M=50.1, S D=10.1)$ and externalizing T-scores $(M=50.3, S D=10.1)$, compared to the non-referred children of the CBCL/1.5-5 norm sample $(M=50.2, S D=$ 10.0 ), and $M=50.2, S D=9.9$, respectively) (Achenbach and Rescorla 2000).

Covariates Potential covariates were selected based on earlier research examining associations of maternal pre- and postnatal anxiety and mindful parenting with child behavioral functioning (Geurtzen et al. 2015; van Batenburg-Eddes et al. 2013). During pregnancy women provided information on demographic and life-style factors (maternal age, parity, education, ethnicity, family income, marital status, prenatal smoking, and alcohol use) by filling in questionnaires. Parity was classified into two categories: 0 versus $\geq 1$ child. Educational level was categorized as high (at least higher vocational training or a bachelor's degree) versus low/medium (lower vocational training or less). Ethnicity was categorized as Dutch or Non-Dutch. Due to small numbers, family income was classified into high ( $>€ 3600$ per month) or (low/below) average, including three categories of family income per month $(<€ 1500, € 1600$ to $€ 2100$ or $€ 2200$ to $€ 3500)$. Information on birth weight, gestational age, and child sex was obtained from medical records filled in by gynecologists and midwives at birth.

To assess infant negative affectivity at age 3 months $\left(M_{\text {months }}=3.3, S D=1.5\right)$ using maternal report, the negative affectivity subscale of the Dutch very short version of the revised Infant Behavior Questionnaire was used (IBQR) (Gartstein and Rothbart 2003). The negative affectivity scale comprises 13 items using a 7-point Likert scale ( $1=$ never, $7=$ always). Mothers filling in this subscale were asked to report on the frequency of certain infant behaviors in specific situations of the previous week, such as "When tired, how often did your baby show distress?" In this study, the internal consistency of the negative affectivity subscale was good $\alpha=0.87$.

\section{Data Analyses}

Demographic and perinatal variables that were correlated with the potential mediators (maternal state and general anxiety at age 4 years, and mindful parenting) and child outcome were selected as covariates. Based on exploratory analyses, we only selected maternal educational level as a covariate, as it was significantly correlated with maternal state anxiety at age 4 years, $r(116)=0.18, p=.049$. The other potential covariates were not significantly correlated with the possible mediators and child outcome. First, we tested whether measures of maternal state and general anxiety during pregnancy and early childhood and mindful parenting were related to each other and to child behavioral/emotional problems using Pearson correlations. According to Cohen (1988), rs $<0.3$ are considered small effects, $0.3>\mathrm{rs}<0.5$ moderate effects, and $\mathrm{rs}>0.5$ large effects.

Second, based on significant correlations identified, we then examined whether maternal (state and general) anxiety and mindful parenting at age 4 years were single mediators of the possible associations between maternal state or general anxiety during pregnancy and child internalizing or externalizing problems using Model 4 of the SPSS macro PROCESS (Hayes 2013). PROCESS uses the bootstrap method to analyze (moderated) mediation models to estimate the indirect effect of one or more potential mediator(s) using 10,000 resamples and calculating bias-corrected bootstrap 95\% confidence intervals (CIs) (Hayes 2013). This approach tends to be more reliable for estimations based on a small sample like the current one (Hayes and Scharkow 2013) and accounts for the possibility of non-normality and/or asymmetry of indirect effects (Hayes 2013). To indicate effect sizes of the tested mediation models, we report completely standardized indirect effects $(\beta)$. Indirect effects are considered significant when CIs do not contain zero.

Third, variables that were shown to be significant single mediators were simultaneously entered into multiple sequential mediation models using Model 6 of PROCESS. Model 6 was used to examine which of these variables were independent mediators of these links and whether the maternal anxiety variables and mindful parenting mediate these links in a sequential manner. Collinearity can attenuate the effects of the mediators in multiple mediator models in a similar way as in multiple linear regression (Preacher and Hayes 2008). To avoid collinearity but also to be able to present results for both types of anxiety, mediation analyses were run separately for state and general anxiety per measurement period. For example, maternal state and general anxiety at age 4 years were strongly correlated, $r(116)=.733, p<.001)$. Finally, Model 14 of PROCESS was used to test whether child sex moderated the possible mediation effects of the concurrent maternal anxiety variables and mindful 
Table 2 Correlations between maternal state and general anxiety during pregnancy at age 4 years, mindful parenting, and child behavioral/emotional problems at age 4 years $(\mathrm{N}=118)$

\begin{tabular}{|c|c|c|c|c|c|c|c|}
\hline Variable & 1 & 2 & 3 & 4 & 5 & 6 & 7 \\
\hline 1. State anxiety at 21 weeks' gestation & & $0.668 * * *$ & $0.378^{* *}$ & $0.237 * *$ & $0.218^{*}$ & $0.211 *$ & 0.002 \\
\hline 2. General anxiety at 21 weeks' gestation & & & $0.223^{*}$ & $0.319 * *$ & -0.142 & $0.240^{*}$ & $0.206^{*}$ \\
\hline 3. State anxiety at age 4 years & & & & $0.733 * * *$ & $-0.578 * * *$ & $0.361 * *$ & $0.209 * *$ \\
\hline 4. General anxiety at age 4 years & & & & & $-0.393 * *$ & $0.225^{*}$ & 0.127 \\
\hline 5. Mindful parenting & & & & & & $-0.331 * *$ & $-0.195^{*}$ \\
\hline 6. Child internalizing problems & & & & & & & $0.626 * * *$ \\
\hline \multicolumn{8}{|l|}{ 7. Child externalizing problems } \\
\hline$M$ & 31.2 & 12.9 & 33.1 & 13.1 & 115.9 & 7.1 & 10.5 \\
\hline$S D$ & 7.1 & 3.2 & 9.4 & 4.1 & 10.1 & 5.8 & 6.5 \\
\hline
\end{tabular}

$* p<.05 ; * * p<.01 ; * * * p<.001$

parenting on the link between maternal (state or general) anxiety during pregnancy and child outcome at age 4 years.

\section{Results}

Descriptives Table 2 shows correlations between maternal measures of prenatal maternal anxiety and, assessed at age 4 years, concurrent maternal anxiety, mindful parenting, and child behavioral/emotional problems. Maternal state anxiety during pregnancy was negatively correlated with mindful parenting but positively with concurrent general anxiety and child internalizing problems at age 4 years. General anxiety during pregnancy was positively associated with concurrent state and general anxiety and with both child outcomes but not with mindful parenting. Mindful parenting was negatively correlated with both child outcomes. Concurrent general and state anxieties were negatively associated with mindful parenting and positively with child internalizing problems. Concurrent state anxiety but not concurrent general anxiety was positively correlated with child externalizing problems.

Single Mediator Models Based on the significant correlations identified, we tested three single mediator models, which were controlled for maternal educational level (see Table 3). The first two single mediator models showed that concurrent maternal state and general anxiety were each significant single mediators of the link of maternal state anxiety during pregnancy with child internalizing problems, $\beta=0.115,95 \% \mathrm{CI}$ $[0.035,0.225]$, and $\beta=0.040,95 \%$ CI $[0.002,0.102]$, respectively (see Table 3 for more detail). Also, mindful parenting was a significant single mediator of the association between maternal state anxiety during pregnancy and child internalizing problems, $\beta=0.060,95 \%$ CI $[0.008,0.148]$ (see Fig. 1). In this single mediator model, maternal state anxiety during pregnancy was negatively related to mindful parenting, $\mathrm{B}=-$
$0.29,95 \%$ CI $[-0.55,-0.04], t(115)=3.21, p=.025$, which was, in turn, related to lower levels of child internalizing problems, $\mathrm{B}=-0.16,95 \%$ CI $[-0.27,-0.06], t(114), p=.002$. Before entering mindful parenting into the model, maternal state anxiety during pregnancy was associated with more child internalizing problems, $\mathrm{B}=0.16,95 \% \mathrm{CI}[0.02,0.31], t(115)$, $p=.03$. However, after adding mindful parenting to the model, maternal state anxiety during pregnancy was no longer directly related to child internalizing problems, $\mathrm{B}=0.11$, $95 \% \mathrm{CI}[-0.03, .26], t(114)=1.56, p=.12$.

Table 3 Indirect effects of concurrent maternal general and state anxiety and mindful parenting on maternal anxiety during pregnancy and child internalizing problems at age 4 years $(N=118)$

\begin{tabular}{|c|c|c|c|c|}
\hline Mediation model & Estimate & $95 \% \mathrm{CI}$ & $t(115)$ & $p$ \\
\hline \multicolumn{5}{|c|}{$\begin{array}{l}\text { Prenatal state anxiety } \rightarrow \text { concurrent state anxiety } \rightarrow \text { child internalizing } \\
\text { problems }\end{array}$} \\
\hline Total effect & 0.161 & {$[0.015,0.308]$} & 2.18 & .03 \\
\hline Direct effect & 0.068 & {$[-0.083,0.219]$} & 0.89 & .37 \\
\hline Indirect effect & 0.115 & {$[0.035 ; 0.225]$} & n.a. & n.a. \\
\hline \multicolumn{5}{|c|}{$\begin{array}{l}\text { Prenatal state anxiety } \rightarrow \text { concurrent general anxiety } \rightarrow \text { child internalizing } \\
\text { problems }\end{array}$} \\
\hline Total effect & 0.161 & {$[0.015,0.308]$} & 2.18 & .03 \\
\hline Direct effect & 0.129 & {$[-0.020,0.277]$} & 1.71 & .09 \\
\hline Indirect effect & 0.040 & {$[0.002 ; 0.102]$} & n.a. & n.a. \\
\hline \multicolumn{5}{|c|}{$\begin{array}{l}\text { Prenatal state anxiety } \rightarrow \text { mindful parenting } \rightarrow \text { child internalizing } \\
\text { problems }\end{array}$} \\
\hline Total effect & 0.161 & {$[0.015,0.308]$} & 2.18 & .03 \\
\hline Direct effect & 0.113 & {$[-0.031,0.257]$} & 1.56 & .12 \\
\hline Indirect effect & 0.060 & {$[0.008 ; 0.148]$} & n.a. & n.a. \\
\hline
\end{tabular}

Estimates were derived from single mediator models based on the bootstrap method (Hayes 2013). Indirect effects are based on standardized estimates

$C I$ bias-corrected confidence interval of single mediator models controlled for maternal educational level, n.a. not applicable 


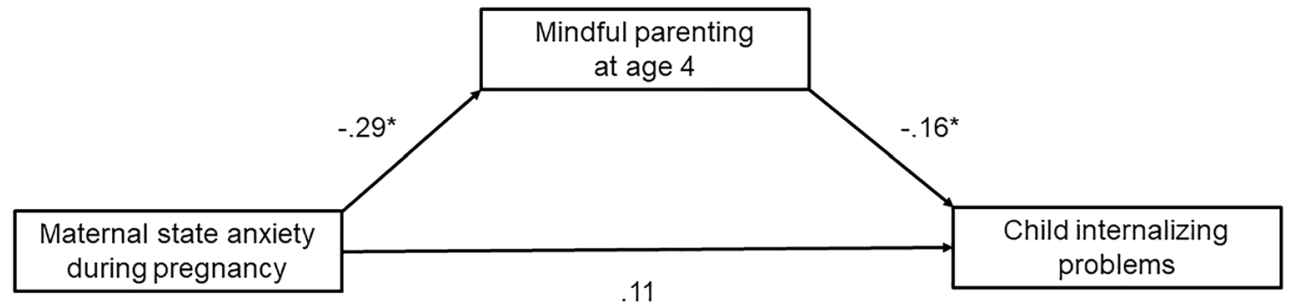

Fig. 1 Single mediator model showing the association of maternal state anxiety during pregnancy and child internalizing problems at age 4 years via mindful parenting controlled for maternal educational level

Multiple Sequential Mediation Models As the two concurrent maternal anxiety constructs and mindful parenting were shown to be significant single mediators, two multiple mediation models assessed the unique contribution of these variables on the link between maternal state anxiety during pregnancy and child internalizing problems at age 4 years. These analyses were conducted separately for concurrent maternal state anxiety and concurrent general anxiety to avoid collinearity. Maternal concurrent general anxiety and mindful parenting sequentially mediated the link between maternal state anxiety during pregnancy and child internalizing problems, $\beta=0.021,95 \%$ CI $[0.002,0.060]$ (see Fig. 2). However, in this model, no independent indirect effects on this link via, respectively, concurrent maternal general anxiety, $\beta=0.019$, $95 \%$ CI $[-0.022,0.076]$, and mindful parenting, $\beta=0.032$, $95 \%$ CI $[-0.002 ; 0.114]$, were observed. Maternal state anxiety during pregnancy was not directly linked to child internalizing problems at age 4 years, $\mathrm{B}=0.10 ; 95 \%$ CI $[-0.04$, $0.25], t(113)=1.39, p=.16$. When entering concurrent maternal state anxiety into the multiple mediation model instead of concurrent maternal general anxiety, both concurrent maternal state anxiety, $\beta=0.07895 \%$ CI $[0.000,0.188]$, and mindful parenting, $\beta=-0.002,95 \%$ CI $[-0.339,0.209]$ were not independent mediators of this link. Also, the sequential mediation effect via concurrent maternal state anxiety and mindful parenting in early childhood was non-significant, $\beta=0.038$, $95 \%$ CI $[-0.008,0.106]$.

Child Sex Finally, we added child sex to the two multiple mediation models to test whether it moderated mediation relationships between prenatal maternal state anxiety, concurrent maternal (state or general) anxiety, mindful parenting, and child internalizing problems at age 4 years. In the first model, indexes of moderated mediation showed that child sex did not moderate the indirect effect of prenatal maternal state anxiety on child internalizing problems via maternal concurrent general anxiety, $\mathrm{B}=-0.087,95 \% \mathrm{CI}[-0.275,0.045]$, and mindful parenting. The second model, including concurrent general anxiety, $\mathrm{B}=0.004,95 \% \mathrm{CI}[-0.105,0.106]$, and mindful parenting, $\mathrm{B}=0.026,95 \% \mathrm{CI}[-0.039,0.141]$ as possible mediators, also revealed non-significant indexes of moderated mediation.

\section{Discussion}

This cohort study showed that the association between maternal anxiety during pregnancy and child internalizing problems at age 4 years was mediated by concurrent maternal general anxiety and, in turn, by mindful parenting. Mindful parenting only mediated this association when concurrent general anxiety was included in the sequential multiple mediation model, but not when concurrent state anxiety was included. Mediators of the association between maternal anxiety during pregnancy and child externalizing problems were not identified. The mediating effects of maternal general anxiety in early childhood and mindful parenting on the link between maternal state anxiety during pregnancy and child internalizing problems were not moderated by child sex.

Our findings extend previous work within the field of research into maternal psychological distress/anxiety during pregnancy and child development by introducing the concept of mindful parenting. According to Duncan et al. (2009), the promotion of mindful parenting skills can improve the quality of parent-child relationships. In a randomized pilot intervention trial, a mindful parenting program had significantly stronger positive effects on mindful parenting, child management skills, and the quality of the parent-child relationship than a

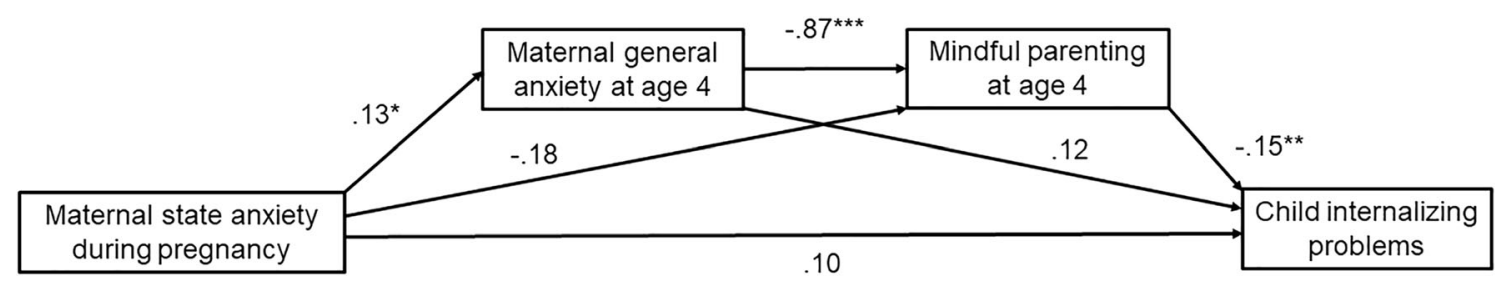

Fig. 2 Sequential multiple mediation model of the association between maternal state anxiety in pregnancy and child internalizing problems at age 4 years via concurrent maternal general anxiety and, in turn, via mindful parenting controlled for maternal educational level 
standard parenting program (Coatsworth et al. 2010). Also, previous work showed that mindful parenting is associated with less child behavioral/emotional problems (Geurtzen et al. 2015; Parent et al. 2016). Our study illustrated the importance of mindful parenting for early child behavioral development in the prenatal psychological distress field. Maternal anxiety during pregnancy was associated with poorer mindful parenting via maternal general anxiety at age 4 years. Our results provide some evidence that poor mindful parenting is, in turn, linked to child internalizing problems at age 4 years.

Interestingly, mindful parenting was no longer a significant mediator when concurrent maternal state anxiety was included in the sequential multiple mediation model instead of concurrent general anxiety. This may simply indicate that mindful parenting is not an independent mediator of the link between maternal anxiety during pregnancy and child internalizing problems at age 4 years (when adding concurrent state anxiety to the model). Concurrent maternal state anxiety was more strongly correlated with mindful parenting than it was with concurrent general anxiety. One might speculate that a common deficit, i.e., worry, might underlie state anxiety and poor mindful parenting. Worry is a key component of state anxiety and has been shown to impair parenting abilities (Choi et al. 2010; Flett et al. 1994). Future studies should examine whether worry or other state anxiety-specific cognitive or emotional factors account for the links between maternal state anxiety in pregnancy and early childhood, mindful parenting, and child internalizing problems.

In comparison to child internalizing problems, child externalizing problems were less consistently associated with maternal state and general anxiety during pregnancy as well as at age 4 years. Similarly, van Batenburg-Eddes et al. (2013) reported that maternal anxiety during pregnancy was linked to a higher risk for child emotional problems at age 3 and 4 years but not with child attention problems. These findings are not surprising since anxiety is an internalizing problem that is transmitted across generations via genetic and environmental influences (Stein et al. 2014).

Child sex did not moderate the mediating effects of concurrent maternal anxiety and mindful parenting on the link between maternal state anxiety during pregnancy and child internalizing problems at age 4 years. This study possibly lacked the statistical power needed to detect such moderated mediation effects, or our results may suggest that child sex is simply not a moderator of these mediation effects.

As maternal anxiety during pregnancy continued into early childhood in the current study, one might speculate that maternal anxiety affects the development of parenting skills from early life onwards. The emotional tie that a mother develops with her unborn child throughout pregnancy lays the foundation for subsequent mother-child interactions and parenting behavior (Brandon et al. 2009; Hicks et al. 2018; Van den
Bergh and Simons 2009). From pregnancy onwards, anxious mothers may have difficulties forming healthy emotional bonds with their (unborn) children (Brandon et al. 2009). These difficulties may continue into early childhood and may together with maternal anxiety negatively affect the development of a mother's mindful parenting abilities and, in turn, the relationship with her child (Brandon et al. 2009; de Cock et al. 2016; Goodman and Gotlib 1999; Stein et al. 2014). It has been hypothesized that maternal anxiety can affect parenting via altered parental cognitions, emotions, and behavior (Goodman and Gotlib 1999). Rumination and emotional distress concern important features of anxiety that have been shown to decrease a mother's ability to attend to, and be responsive to, her child (Stein et al. 2012). Parental attention and responsiveness are crucial elements of mindful parenting (Bögels et al. 2010). Future research should investigate whether maternal anxiety negatively influences mindful parenting via rumination and emotional distress as well as via alterations in parental attention and responsiveness.

\section{Mechanisms and Mediating Factors}

Our findings provide some support for the idea that maternal anxiety and mindful parenting in early childhood are mediators in the link between maternal state anxiety during pregnancy and child internalizing problems. Maternal anxiety during pregnancy, maternal postnatal anxiety, and poor parenting in early childhood have all been previously shown to be related to children's neurodevelopmental problems and behavioral/emotional problems (O'Connor et al. 2002; Stein et al. 2014; Van den Bergh et al. 2017). Considering the existing empirical evidence and conceptual models, it is tempting to speculate that alterations in child (neuro) behavioral patterns due to exposure to prenatal maternal anxiety may progress over time and result in long-lasting behavioral deficits through bidirectional interactions with adverse (postnatal) environmental factors, such as maternal anxiety and poor mindful parenting (Klahr and Burt 2014; Rutherford et al. 2015; Stein et al. 2014). Future studies should examine these possible bidirectional effects from early life onwards using longitudinal cross-lagged models. Interestingly, the parental genetic makeup has a significant impact on parenting behavior (Klahr and Burt 2014). Also, anxiety and behavioral problems have a common genetic basis (Plomin et al. 1994; Clement et al. 2002). Shared genetic factors may, therefore, also partly account for the links between maternal anxiety, mindful parenting, and child internalizing problems.

\section{Strengths and Limitations}

Strengths of this study include its prospective design, the follow-up until age 4 years, and the assessment of possible psychosocial risk and protective factors, including 
anxiety and mindful parenting. Yet, this study also has several potential limitations. Firstly, data were based on maternal reports. Anxious mothers may be more likely to overreport child behavioral/emotional problems. Yet, there is doubt whether mothers with psychological problems have distorted perceptions of their children's problems (Richters 1992). Behavioral/emotional problems of children aged 2-3 years and of children and adolescents aged 4-16 years as reported by parents using the CBCL were predictive of DSM-IV diagnosed psychopathological counterparts 8 and 24 years later, respectively (Mesman and Koot 2001; Reef et al. 2010). Secondly, this study does not completely allow us to draw inferences about the causality and directionality of effects based on the tested mediation models, because data on the mediators and outcomes were collected cross-sectionally. All the same, previous research supports the theoretical assumptions of the tested mediation model as mindful parenting and maternal anxiety are interrelated and have both been shown to be related with child behavioral/emotional problems (Barker et al. 2011; Meppelink et al. 2016; Parent et al. 2016). Thirdly, the sample size of this study is relatively small. Yet, using the bias-corrected bootstrap CI may have partly overcome this matter, as this procedure is more likely to result in correct statistical decisions than any other statistical method regardless of sample size (Hayes and Scharkow 2013). Finally, selection factors might have reduced the associations between maternal state anxiety during pregnancy, the mediators, and child outcome. Maternal educational and family income levels were particular high as well as the rate of Dutch ethnicity. Data on child outcomes were more complete in mothers with lower levels of maternal anxiety during pregnancy.

Acknowledgements We gratefully acknowledge the contribution of participating families and students supporting the data collection. The PELS study is supported by the national funding agencies of the European Science Foundation participating in the EUROCORES EuroSTRESS Programme, i.e., the Brain and Cognition Program of the Netherlands Organization for Scientific Research for the Netherlands ((http:// archives.esf.org/ coordinating-research/eurocores/ programmes/ eurostress/projects/ pels.html). BVdB is project leader of the PELS study conducted in collaboration with V. Glover (Imperial College London), S. Claes (KU Leuven), and A. Rodriguez (Uppsala University).

Author Contributions JH contributed to the study design, conducted data analyses and wrote the paper. MIvdH and ABW contributed to the study design, interpreting the results, and writing the paper. MIvdH performed data collection. JW provided statistical advice, interpreted and edited the results and data analyses section. BVdB designed the study and collaborated in interpreting results and writing the paper.

Funding information $\mathrm{BVdB}$ is supported by the European Commission 7th Framework Programme (FP7-HEALTH. 2011.2.2.2-2 BRAINAGE, Grant no. 279281).

\section{Compliance with Ethical Standards}

All procedures performed in studies involving human participants were in accordance with the ethical standards of the institutional and/or national research committee and with the 1964 Helsinki declaration and its later amendments or comparable ethical standards. The Medical Ethical Committee of St. Elisabeth Hospital Tilburg, the Netherlands approved the PELS study. The Ethical Review Board of Tilburg University approved its 4-year-follow-up.

Conflict of Interest The authors declare that they have no conflict of interest.

Informed Consent Informed consent was obtained from all individual participants included in the study.

Open Access This article is distributed under the terms of the Creative Commons Attribution 4.0 International License (http:// creativecommons.org/licenses/by/4.0/), which permits unrestricted use, distribution, and reproduction in any medium, provided you give appropriate credit to the original author(s) and the source, provide a link to the Creative Commons license, and indicate if changes were made.

Publisher's Note Springer Nature remains neutral with regard to jurisdictional claims in published maps and institutional affiliations.

\section{References}

Achenbach, T. M., \& Rescorla, L. A. (2000). Manual for ASEBA preschool forms \& profiles. Burlington, VT: University of Vermont, Research Center for Children, Youth and Families.

Arrindell, W. A., \& Ettema, J. H. M. (2003). SCL-90. Symptom checklist. In Swets Test Publishers.

Barker, E. D., Jaffee, S. R., Uher, R., \& Maughan, B. (2011). The contribution of prenatal and postnatal maternal anxiety and depression to child maladjustment. Depression and Anxiety, 28(8), 696-702. https://doi.org/10.1002/da.20856.

Barnett, M. A., \& Scaramella, L. V. (2013). Mothers' parenting and child sex differences in behavior problems among African American preschoolers. Journal of Family Psychology, 27(5), 773-783. https:// doi.org/10.1037/a0033792.

Beijers, R., Buitelaar, J. K., \& de Weerth, C. (2014). Mechanisms underlying the effects of prenatal psychosocial stress on child outcomes: beyond the HPA axis. European Child \& Adolescent Psychiatry, 23(10), 943-956. https://doi.org/10.1007/s00787-014-0566-3.

Bergink, V., Kooistra, L., Lambregtse-van den Berg, M. P., Wijnen, H., Bunevicius, R., van Baar, A., \& Pop, V. (2011). Validation of the Edinburgh depression scale during pregnancy. Journal of Psychosomatic Research, 70(4), 385-389. https://doi.org/10.1016/ j.jpsychores.2010.07.008.

Bögels, S. M., Lehtonen, A., \& Restifo, K. (2010). Mindful parenting in mental health care. Mindfulness, 1(2), 107-120. https://doi.org/10. 1007/s12671-010-0014-5.

Brandon, A. R., Pitts, S., Denton, W. H., Stringer, C. A., \& Evans, H. M. (2009). A history of the theory of prenatal attachment. Journal of Prenatal \& Perinatal Psychology \& Health, 23(4), 201-222.

Choi, H., Yamashita, T., Wada, Y., Narumoto, J., Nanri, H., Fujimori, A., et al. (2010). Factors associated with postpartum depression and abusive behavior in mothers with infants. Psychiatry and Clinical Neurosciences, 64(2), 120-127. https://doi.org/10.1111/j.14401819.2010.02063.x. 
Clark, L. A., \& Watson, D. (1991). Tripartite model of anxiety and depression: psychometric evidence and taxonomic implications. Journal of Abnormal Psychology, 100(3), 316-336.

Clément, Y., Calatayud, F., \& Belzung, C. (2002). Genetic basis of anxiety-like behaviour: a critical review. Brain Research Bulletin, 57(1), 57-71.

Coatsworth, J. D., Duncan, L. G., Greenberg, M. T., \& Nix, R. L. (2010). Changing parent's mindfulness, child management skills and relationship quality with their youth: Results from a randomized pilot intervention trial. Journal of Child and Family Studies, 19(2), 203-217.

Dayton, C. J., Levendosky, A. A., Davidson, W. S., \& Bogat, G. A. (2010). The child as held in the mind of the mother: the influence of prenatal maternal representations on parenting behaviors. Infant Mental Health Journal, 31(2), 220-241. https://doi.org/10.1002/ imhj.20253.

de Bruin, E. I., Zijlstra, B. J. H., Geurtzen, N., van Zundert, R. M. P., van de Weijer-Bergsma, E., Hartman, E. E., et al. (2014). Mindful parenting assessed further: psychometric properties of the Dutch version of the interpersonal mindfulness in parenting scale (IM-P). Mindfulness, 5(2), 200-212. https://doi.org/10.1007/s12671-0120168-4.

de Cock, E. S. A., Henrichs, J., Vreeswijk, C. M. J. M., Maas, A. J. B. M., Rijk, C. H. A. M., \& van Bakel, H. J. A. (2016). Continuous feelings of love? The parental bond from pregnancy to toddlerhood. Journal of Family Psychology, 30(1), 125-134. https://doi.org/10.1037/ fam0000138.

Derogatis, L. R., Lipman, R. S., Rickels, K., Uhlenhuth, E. H., \& Covi, L. (1974). The Hopkins symptom checklist (HSCL) a measure of primary symptom dimension. In P. Pichot (Ed.), Psychological measurements in psychopharmacology (pp. 79-110). Basel: Karger.

Duncan, L. G., Coatsworth, J. D., \& Greenberg, M. T. (2009). A model of mindful parenting: implications for parent-child relationships and prevention research. Clinical Child and Family Psychology Review, 12(3), 255-270. https://doi.org/10.1007/s10567-009-0046-3.

Edwards, R. C., \& Hans, S. L. (2016). Prenatal depressive symptoms and toddler behavior problems: the role of maternal sensitivity and child sex. Child Psychiatry \& Human Development, 47(5), 696-707. https://doi.org/10.1007/s10578-015-0603-6.

Flett, G. L., Endler, N. S., Tassone, C., \& Hewitt, P. L. (1994). Perfectionism and components of state and trait anxiety. Current Psychology, 13(4), 326-350. https://doi.org/10.1007/BF02686891.

Gartstein, M. A., \& Rothbart, M. K. (2003). Studying infant temperament via the revised infant behavior questionnaire. Infant Behavior and Development, 26(1), 64-86. https://doi.org/10.1016/S01636383(02)00169-8.

Gelenberg, A. J. (2000). Psychiatric and somatic markers of anxiety: identification and pharmacologic treatment. Primary Care Companion to the Journal of Clinical Psychiatry, 2(2), 49-54.

Geurtzen, N., Scholte, R. H. J., Engels, R. C. M. E., Tak, Y. R., \& van Zundert, R. M. P. (2015). Association between mindful parenting and adolescents' internalizing problems: non-judgmental acceptance of parenting as core element. Journal of Child and Family Studies, 24(4), 1117-1128. https://doi.org/10.1007/s10826-0149920-9.

Goodman, S. H., \& Gotlib, I. H. (1999). Risk for psychopathology in the children of depressed mothers: a developmental model for understanding mechanisms of transmission. Psychological Review, 106(3), 458-490. https://doi.org/10.1037/0033-295X.106.3.458.

Hayes, A. F. (2013). Introduction to mediation, moderation, and conditional process analysis : a regression-based approach. New York, NY: Guilford Press.

Hayes, A. F., \& Scharkow, M. (2013). The relative trustworthiness of inferential tests of the indirect effect in statistical mediation analysis. Psychological Science, 24(10), 1918-1927. https://doi.org/10.1177/ 0956797613480187.
Henrichs, J., Rescorla, L., Donkersloot, C., Schenk, J. J., Raat, H., Jaddoe, V. W. V., et al. (2013). Early vocabulary delay and behavioral/emotional problems in early childhood: the generation $\mathrm{R}$ study. Journal of Speech Language and Hearing Research, 56(2), 553. https://doi.org/10.1044/1092-4388(2012/11-0169.

Henrichs, J., Schenk, J. J., Schmidt, H. G., Velders, F. P., Hofman, A., Jaddoe, V. W. V., et al. (2009). Maternal pre- and postnatal anxiety and infant temperament. the generation $\mathrm{R}$ study. Infant and Child Development, 18(6), 556-572. https://doi.org/10.1002/icd.639.

Hicks, L. M., Dayton, C. J., Brown, S., Muzik, M., \& Raveau, H. (2018). Mindfulness moderates depression and quality of prenatal attachment in expectant parents. Mindfulness, 9(5), 1604-1614. https:// doi.org/10.1007/s12671-018-0907-2.

Hofstra, M. B., Van der Ende, J., \& Verhulst, F. C. (2000). Continuity and change of psychopathology from childhood into adulthood: a 14year follow-up study. Journal of the American Academy of Child \& Adolescent Psychiatry, 39(7), 850-858. https://doi.org/10.1097/ 00004583-200007000-00013.

Junge, C., Garthus-Niegel, S., Slinning, K., Polte, C., Simonsen, T. B., \& Eberhard-Gran, M. (2017). The impact of perinatal depression on children's social-emotional development: a longitudinal study. Maternal and Child Health Journal, 21(3), 607-615. https://doi. org/10.1007/s10995-016-2146-2.

Klahr, A. M., \& Burt, S. A. (2014). Elucidating the etiology of individual differences in parenting: a meta-analysis of behavioral genetic research. Psychological Bulletin, 140(2), 544-586. https://doi.org/10. 1037/a0034205.

Koelewijn, J. M., Sluijs, A. M., \& Vrijkotte, T. G. M. (2017). Possible relationship between general and pregnancy-related anxiety during the first half of pregnancy and the birth process: a prospective cohort study. BMJ Open, 7(5), e013413. https://doi.org/10.1136/bmjopen2016-013413.

Kok, R., Bakermans-Kranenburg, M. J., van IJzendoorn, M. H., Velders, F. P., Linting, M., Jaddoe, V. W. V., et al. (2013). The role of maternal stress during pregnancy, maternal discipline, and child COMT Val158Met genotype in the development of compliance. Developmental Psychobiology, 55(5), 451-464. https://doi.org/10. 1002/dev.21049.

Laurent, H. K., Duncan, L. G., Lightcap, A., \& Khan, F. (2017). Mindful parenting predicts mothers' and infants' hypothalamic-pituitaryadrenal activity during a dyadic stressor. Developmental Psychology, 53(3), 417-424. https://doi.org/10.1037/dev0000258.

Maas, A. J. B. M., de Cock, E. S. A., Vreeswijk, C. M. J. M., Vingerhoets, A. J. J. M., \& van Bakel, H. J. A. (2016). A longitudinal study on the maternal-fetal relationship and postnatal maternal sensitivity. Journal of Reproductive and Infant Psychology, 34(2), 110-121. https://doi.org/10.1080/02646838.2015.1112880.

Meppelink, R., de Bruin, E. I., Wanders-Mulder, F. H., Vennik, C. J., \& Bögels, S. M. (2016). Mindful parenting training in child psychiatric settings: heightened parental mindfulness reduces parents' and children's psychopathology. Mindfulness, 7(3), 680-689. https://doi. org/10.1007/s12671-016-0504-1.

Mesman, J., \& Koot, H. M. (2001). Early preschool predictors of preadolescent internalizing and externalizing DSM-IV diagnoses. Journal of the American Academy of Child \& Adolescent Psychiatry, 40(9), 1029-1036. https://doi.org/10.1097/00004583-200109000-00011.

Nieuwenhuijsen, K., de Boer, A. G. E. M., Verbeek, J. H. A. M., Blonk, R. W. B., \& van Dijk, F. J. H. (2003). The depression anxiety stress scales (DASS): detecting anxiety disorder and depression in employees absent from work because of mental health problems. Occupational and Environmental Medicine, 60(suppl 1), i77-i82. https://doi.org/10.1136/OEM.60.SUPPL 1.I77.

O'Connor, T. G., Heron, J., Golding, J., Beveridge, M., \& Glover, V. (2002). Maternal antenatal anxiety and children's behavioural/ emotional problems at 4 years. Report from the Avon Longitudinal Study of Parents and Children. The British Journal of Psychiatry : 
The Journal of Mental Science, 180, 502-508 Retrieved from http:// www.ncbi.nlm.nih.gov/pubmed/12042228.

Parent, J., McKee, L. G., N Rough, J., \& Forehand, R. (2016). The Association of Parent Mindfulness with parenting and youth psychopathology across three developmental stages. Journal of Abnormal Child Psychology, 44(1), 191-202. https://doi.org/10. 1007/s10802-015-9978-x.

Pearson, R. M., Melotti, R., Heron, J., Joinson, C., Stein, A., Ramchandani, P. G., \& Evans, J. (2012). Disruption to the development of maternal responsiveness? The impact of prenatal depression on mother-infant interactions. Infant Behavior and Development, 35(4), 613-626. https://doi.org/10.1016/j.infbeh.2012.07.020.

Plomin, R., Owen, M. J., \& McGuffin, P. (1994). The genetic basis of complex human behaviors. Science (New York, N.Y.), 264(5166), 1733-1739.

Preacher, K. J., \& Hayes, A. F. (2008). Asymptotic and resampling strategies for assessing and comparing indirect effects in multiple mediator models. Behavior Research Methods, 40(3), 879-891. https:// doi.org/10.3758/BRM.40.3.879.

Reef, J., van Meurs, I., Verhulst, F. C., \& van der Ende, J. (2010). Children's problems predict adults' DSM-IV disorders across 24 years. Journal of the American Academy of Child \& Adolescent Psychiatry, 49(11), 1117-1124. https://doi.org/10.1016/j.jaac.2010. 08.002 .

Richters, J. E. (1992). Depressed mothers as informants about their children: a critical review of the evidence for distortion. Psychological Bulletin, 112(3), 485-499.

Rutherford, H. J. V., Wallace, N. S., Laurent, H. K., \& Mayes, L. C. (2015). Emotion regulation in parenthood. Developmental Review, 36, 1-14. https://doi.org/10.1016/j.dr.2014.12.008.

Sandman, C. A., Davis, E. P., Buss, C., \& Glynn, L. M. (2011). Prenatal programming of human neurological function. International Journal of Peptides, 2011, 837596. https://doi.org/10.1155/2011/ 837596.

Siegel, D. J., \& Hartzell, M. (2003). Parenting from the inside out: how a deeper self-understanding can help you raise children who thrive. New York: Penguin.
Spielberger, C. D. (1975). The measurement of state and trait anxiety: conceptual and methodological issues. In L. Levi (Ed.), Emotionstheir parameters and measurement (pp. 713-725). New York: The Raven Press.

Stein, A., Craske, M. G., Lehtonen, A., Harvey, A., Savage-McGlynn, E., Davies, B., et al. (2012). Maternal cognitions and mother-infant interaction in postnatal depression and generalized anxiety disorder. Journal of Abnormal Psychology, 121(4), 795-809. https://doi.org/ 10.1037/a0026847.

Stein, A., Pearson, R. M., Goodman, S. H., Rapa, E., Rahman, A., Mccallum, M., et al. (2014). Perinatal mental health 3: effects of perinatal mental disorders on the fetus and child. The Lancet, 384, 1800-1819. https://doi.org/10.1016/S0140-6736(14)61277-0.

Talge, N. M., Neal, C., \& Glover, V. (2007). Antenatal maternal stress and long-term effects on child neurodevelopment: how and why? Journal of Child Psychology and Psychiatry, 48(3-4), 245-261. https://doi.org/10.1111/j.1469-7610.2006.01714.x.

van Batenburg-Eddes, T., Brion, M. J., Henrichs, J., Jaddoe, V. W. V., Hofman, A., Verhulst, F. C., ... Tiemeier, H. (2013). Parental depressive and anxiety symptoms during pregnancy and attention problems in children: a cross-cohort consistency study. Journal of Child Psychology and Psychiatry, 54(5), 591-600. https://doi.org/ 10.1111 jepp. 12023

Van den Bergh, B. R. H., van den Heuvel, M. I., Lahti, M., Braeken, M., de Rooij, S. R., Entringer, S., et al. (2017). Prenatal developmental origins of behavior and mental health: the influence of maternal stress in pregnancy. Neuroscience \& Biobehavioral Reviews. https://doi.org/10.1016/j.neubiorev.2017.07.003.

Van den Bergh, B., \& Simons, A. (2009). A review of scales to measure the mother-foetus relationship. Journal of Reproductive and Infant Psychology, 27(2), 114-126. https://doi.org/10.1080/ 02646830802007480 .

Van der Ploeg, H. M., Defares, P. B., \& Spielberger, C. D. (1980), Handleiding bij de zelfbeoordelingsvragenlijst [Manual of the State-Trait Anxiety Inventory; Dutch adaptation]. Leiden, the Netherlands: Swets en Zeitlinger. 